\title{
LA EVOLUCIÓN DE LA NUPCIALIDAD EN ESPAÑA: UN ANÁlISIS A TRAVÉS DE LAS ESTADÍSTICAS VITALES Y LOS CENSOS DE POBLACIÓN
}

\author{
POR \\ MARGARITA DELGADO
}

\section{INTRODUCCIÓN}

La nupcialidad es uno de los componentes básicos del análisis demográfico clásico. Junto con la fecundidad extramatrimonial, las tasas de fecundidad marital combinadas con el número de mujeres casadas en edad fecunda, determinan el nivel de fecundidad general o reproducción en una sociedad. Pero no sólo tiene importancia su análisis por la influencia que ejerce sobre la fecundidad, sino que en sí misma es una variable de primer orden por cuanto el matrimonio suele ser el primer paso en el proceso de formación de la familia en el modelo tradicional y hasta hace no muchos años representaba la emancipación de los hijos del hogar paterno. En la actualidad, las vías de emancipación son más diversas y complejas, pero el matrimonio sigue representando - para una gran mayoría - un paso decisivo hacia la vida adulta.

La aparición y difusión en décadas recientes del fenómeno de la cohabitación o parejas «sin papeles» ha modificado de forma significativa el papel jugado por la nupcialidad, por lo que el concepto mismo puede necesitar una redefinición, principalmente en aquellos países en los que las uniones consensuales estables son equiparables a las

Margarita Delgado. Instituto de Economía y Geografía. Consejo Superior de Investigaciones Científicas.

Estudios Geográficos

Tomo LXI, 2000, n. ${ }^{\circ} 241$, octubre-diciembre 
parejas formadas convencionalmente tanto por su influencia en la reproducción, como por la función que desempeñan desde el punto de vista de la socialización del individuo ${ }^{1}$. Ocurre, no obstante, que el fenómeno de las uniones consensuales tiene una incidencia desigual, incluso en contextos sociales similares como pueden ser los países occidentales, ya que mientras en los países del norte de Europa la proporción de parejas que viven en esta situación es elevada, en los del sur, estas nuevas formas de convivencia tienen escasa relevancia.

El matrimonio, además de ser una de las posibles vías hacia el proceso de formación de la familia, constituye un importante factor asociado a los movimientos migratorios, por cuanto puede desencadenar movimientos intra e interprovinciales para uno o ambos cónyuges, así como cambios de residencia intramunicipales relacionados con la oferta/demanda de viviendas. Asimismo, es una de las variables demográficas más sensibles a la coyuntura económica. Su curso varía en consonancia con la situación favorable o desfavorable de la economía, por cuanto es una decisión que los individuos suelen tomar una vez que han logrado una posición en el mercado de trabajo y tienen ciertas expectativas de continuidad. Por ello, es un evento cuyo calendario está muy determinado por factores tales como el empleo y las condiciones de acceso a la vivienda.

La vinculación de la nupcialidad a la situación económica es una constante histórica en España. Ya en épocas pasadas, cuando la sociedad era fundamentalmente agraria, la posibilidad de contraer matrimonio estaba determinada - en buena medida - por el acceso a la propiedad de la tierra ${ }^{2}$. En consonancia con ello, a lo largo de este siglo los indicadores nupciales reflejan claramente las adversas condiciones de los años de la posguerra, así como la bonanza de la década de los sesenta. Igualmente, llegados a los noventa, las dificultades por las que atraviesa el mercado de trabajo y el alto índice de desempleo juvenil, se traducen en un claro retraso y baja intensidad del matrimonio (Miret-Gamundi, 1997). Ello implica que los jóvenes dilatan el período de convivencia con los padres (Fernández Cordón, 1997) y retrasan su integra-

\footnotetext{
${ }^{1}$ Una temprana consideración acerca de la nueva óptica con que habrán de contemplarse las uniones consensuales se encuentra en Coale, A. J. and Treadway, R. (1986).

${ }^{2}$ Véase Livi Bacci, M. (1968), donde se señala el sistema de propiedad de la tierra como determinante del matrimonio más o menos tardío. También Cachinero Sánchez, B. (1981).
} 
ción en la vida adulta. Si bien en otros países europeos el descenso y retraso de la nupcialiad se acompañan de un importante incremento de las uniones consensuales, en el caso español la cohabitación muestra un aumento muy moderado (Delgado y Castro Martín, 1998), como moderado resulta, asimismo, el aumento de la fecundidad fuera del matrimonio. Las tendencias recientes muestran, asimismo, una disminución de las concepciones prenupciales (Muñoz Pérez, 1995) y un aumento de la ruptura matrimonial (Alberdi, 1999).

El propósito de este trabajo consiste en analizar la evolución reciente de la nupcialidad desde distintas ópticas, utilizando fuentes, indicadores y métodos diversos, resaltando las ventajas y limitaciones de cada uno. El estudio se centra en la evolución observada en las dos últimas décadas, pero tratando de encuadrarla en el contexto precedente, tanto europeo como español. También se examinan las diferencias existentes entre comunidades autónomas.

\section{Fuentes Y METODOLOGÍA}

El análisis de la nupcialidad y la construcción de los indicadores que muestran su evolución puede abordarse a partir de diferentes fuentes, las cuales determinan tanto el enfoque como la metodología a emplear. En este trabajo se utilizan dos de las principales fuentes disponibles para el análisis cuantitativo de las pautas matrimoniales: las estadísticas vitales y los recuentos de población ${ }^{3}$.

\section{Estadísticas vitales}

El Movimiento Natural de la Población (M.N.P.) constituye una de las principales fuentes para estudiar las pautas de nupcialidad, por cuanto proporciona el número de matrimonios que se celebran en un año dado

${ }^{3}$ No se han utilizado las estadísticas de ruptura matrimonial (separación y divorcio) que publica el Consejo General del Poder Judicial en sus Memorias Anuales, debido a la escasa información que ofrecen dichos datos. En ellas sólo se indica si la separación o divorcio se ha producido con o sin acuerdo, pero no otros datos que serían de gran interés, tales como la edad de los divorciados, la duración del matrimonio o el número de hijos si los hubiere, por citar sólo los más relevantes. 
por sexo, edad, estado civil anterior de los contrayentes, lugar de residencia, cohorte de nacimiento, forma de la celebración - matrimonio civil o religioso, incluyendo el rito por el que se ha celebrado-, entre otras clasificaciones $^{4}$.

En España, el M.N.P. presenta algunas deficiencias para el estudio de la evolución de la nupcialidad debido al subregistro de matrimonios que se constata entre 1980 y 1986 . Tras la revisión del Concordato entre la Santa Sede y el Estado español a fines de los años setenta, al matrimonio canónico se le reconocieron efectos civiles. A partir de ese momento, los párrocos enviaban los boletines eclesiásticos de matrimonio al Instituto Nacional de Estadística, pero no en todas las diócesis estos envíos tenían un carácter exhaustivo, por lo que empezó a detectarse un cierto subregistro. Las cifras totales de matrimonios están infraestimadas para el período $1980-1986^{5}$, aunque por medio de las estadísticas de la propia Iglesia Católica ha podido estimarse y corregirse dicho subregistro para el Total Nacional, no así para las provincias al no coincidir la demarcación civil con la eclesiástica. El subregistro fue corrigiéndose paulatinamente, y desde 1986 se considera que las cifras no adolecen de infraestimación, principalmente debido a que en la actualidad los boletines estadísticos de matrimonio son remitidos al I.N.E. desde el Registro Civil.

En este estudio se van a utilizar como indicadores construidos a partir del M.N.P., las tasas específicas de nupcialidad por edad y el índice sintético de primonupcialidad femenina, así como la edad media de las mujeres al contraer el primer matrimonio.

El índice sintético es una medida resumen de la intensidad de la nupcialidad, pues es el resultado de la suma de las tasas específicas de primera nupcialidad por edades simples de 15 a 49 años. En teoría, debería variar de 0 a 1 , ya que equivale al número medio de primeros matrimonios por persona, pero en situaciones de adelanto del calendario nupcial

\footnotetext{
${ }^{4}$ No se han enumerado exhaustivamente todas las clasificaciones que recoge el apartado «matrimonios» del M.N.P. Una descripción de las fuentes demográficas españolas puede encontrarse en Reher, D-S. y Valero Lobo, A. (1995).

${ }^{5}$ El subregistro que se ha detectado entre 1980-1986 para el Total Nacional varía entre el $2 \%$ y el 18\%, alcanzando en 1982 y 1983 su cifra más elevada, con $17 \%$ y 18\% respectivamente. Hay que hacer notar que, territorialmente, el subregistro en absoluto es homogéneo. Para una explicación más detallada del problema, así como de las provincias más afectadas, y discusión del procedimiento empleado en la corrección, véase Delgado Pérez, M. y Fernández Cordón, J. A. (1989).
} 
puede llegar a superar la unidad, algo a primera vista un tanto ilógico, pero que refleja un cierto «boom» de matrimonios, producto de un rejuvenecimiento, cuando se calcula para una cohorte ficticia en un año concreto. Calculado en la forma habitual - primeros matrimonios a una determinada edad en el numerador y el total de mujeres de esa edad en el denominador- está afectado por la estructura de la población por estado civil, determinada por la nupcialidad experimentada por cada grupo de edad en un momento previo. A fin de contar con un indicador que elimine este efecto, se han calculado tasas de primera nupcialidad femenina, cuyo denominador son las mujeres solteras en cada grupo de edad ${ }^{6}$.

El principal inconveniente de una medida resumen como es el índice sintético de primonupcialidad es que no permite distinguir los efectos de intensidad y calendario. Un descenso del índice puede deberse a un simple retraso de la nupcialidad, o puede ser un descenso real de la intensidad, o ambas cosas simultáneamente. Son problemas inherentes a las medidas sintéticas transversales y que, en alguna medida, se tratan de paliar examinando la evolución de las tasas por grupos de edad.

La evolución de la edad media al primer matrimonio permite apreciar los cambios producidos en el calendario de la nupcialidad. La edad a la que se contrae matrimonio tiene gran influencia sobre la fecundidad, pues el efectivo de casados en la población disminuye cuando el matrimonio es más tardío, lo que conlleva que las personas pasen menos años de su vida reproductiva en una unión matrimonial.

\section{Recuentos de población}

Los censos de población y los padrones municipales de habitantes constituyen observaciones transversales periódicas, por lo que sólo proporcionan información «estática» para un momento dado. Sin embargo, es posible inferir la dinámica de las transformaciones en curso a través de los cambios observados en la estructura. Hasta ahora, estos recuentos constituían una importante fuente para el estudio de la nup-

${ }^{6} \mathrm{Al}$ efectivo total en mitad del año se le ha aplicado la proporción de solteras que reflejan los recuentos de población de ese año: 1975, 1981, 1986 y 1991. Para 1995, al no contar con un recuento de población, se ha aplicado la proporción de solteras que refleja la media anual de la Encuesta de Población Activa (E.P.A.). 
cialidad, ya que facilitan una distribución de la población por edad y estado civil. En el padrón de 1996 - último en elaborarse, ya que se abandona este tipo de recuento y se opta por el registro continuo-, desafortunadamente, no se solicitó información sobre el estado civil ${ }^{7}$. Constituye, pues, una laguna en la serie temporal de distribuciones censales y padronales de la población por estado civil, junto con el Censo de 1960, para el que no han sido publicados los datos referidos al Total Nacional, aunque sí los de las provincias.

Los censos de población y padrones de habitantes permiten, mediante un método indirecto ideado por Hajnal (1953), calcular la edad media al primer matrimonio o «singulate mean age at marriage» (SMAM). Este método tiene algunos inconvenientes; entre ellos, interpretar el comportamiento de una cohorte ficticia - construida a partir de las sucesivas generaciones que componen un censo- como si se tratase de una cohorte real. Asimismo, la forma de cálculo de la SMAM asume una población estable y el propio Hajnal advierte que en períodos de cambio en las pautas de nupcialidad, la aproximación resulta menos fiable.

Por otra parte, los efectivos de población en el momento censal están afectados por las pautas de mortalidad a que ha estado sometido cada grupo de edad y por los movimientos migratorios que hayan protagonizado (Cachinero, 1982; Rowland, 1987), lo que pudiera estar influyendo en mayor medida en las unidades de análisis más pequeñas, como son las comunidades autónomas. El inconveniente resulta de menor importancia respecto al Total Nacional.

En este trabajo se va a examinar la evolución seguida desde 1975 por la edad media al matrimonio para hombres y mujeres medida con el indicador SMAM, y comparar esta tendencia, en el caso de las mujeres, con la observada mediante el indicador obtenido a partir del M.N.P. No se busca una exacta equivalencia en el indicador -imposible, por otra parte-, sino una verificación del signo de la tendencia. Las fuentes censales

\footnotetext{
${ }^{7}$ Debido a la entrada en vigor en 1992 de la Ley Orgánica que regula el tratamiento automatizado de datos (LORTAD), sólo se recabarán los datos necesarios para los fines de la indagación que se lleve a efecto. Como el padrón municipal de habitantes es un instrumento de gestión de los ayuntamientos, se considera que el estado civil de los ciudadanos no es estrictamente necesario para los fines a los que responde la elaboración del padrón; motivo por el que no se preguntó en el de 1996. Solamente algunas comunidades introdujeron esta pregunta en la encuesta que realizaron simultáneamente a la recogida de datos padronales.
} 
y las estadísticas vitales tienen la ventaja de que al tratarse de registros exhaustivos, permiten comparaciones detalladas a nivel provincial y de comunidades autónomas, tarea que no siempre se puede hacer con otras fuentes - las encuestas, por ejemplo-, muchas veces representativas sólo a nivel nacional.

\section{ESPAÑA EN EL CONTEXTO EUROPEO}

Desde principios del siglo xx, en la mayoría de los países europeos el modelo tradicional de matrimonio (Hajnal, 1965) - caracterizado por una tardía edad a las primeras nupcias y una alta proporción de célibes definitivos - se fue debilitando. Una de las principales razones de ese debilitamiento fue que el papel que ejercía como elemento de contención del crecimiento demográfico perdió vigencia al iniciarse la transición de la fecundidad, consistente en una reducción de la fecundidad dentro del matrimonio, pasando de una situación de fecundidad natural a otra de fecundidad controlada.

Aunque el abandono del llamado «modelo europeo de matrimonio» es un rasgo generalizado en todo el Continente, los países del sur de Europa acusan un cierto retraso temporal respecto a los del norte en la adopción de las nuevas tendencias. España es un ejemplo de ello, por cuanto el modelo tradicional de nupcialidad se mantuvo vigente hasta mediado el siglo $\mathrm{xx}$, si bien con significativas diferencias regionales (Watkins, 1981). Considerando el conjunto del país, la edad media al primer matrimonio se incrementó durante las primeras décadas del siglo $\mathrm{xx}$ y alcanzó su punto máximo en los años cuarenta. Así, al comienzo del período de posguerra, todavía la elevada edad al casarse y el matrimonio no universal eran rasgos presentes en la sociedad española (Cachinero Sánchez, 1982).

Las mujeres españolas de este siglo muestran claras diferencias en cuanto a la intensidad de su nupcialidad. Observadas longitudinalmente, las generaciones nacidas entre 1900-1915 alcanzaron una proporción final de casadas de sólo el 80\% (Fernández Cordón, 1986). Las últimas cohortes de este subgrupo tenían veinticinco años en torno a 1940, momento en que se registra una elevada edad al matrimonio. La edad media al primer matrimonio, venía incrementándose desde 1887 - año del primer censo en el que aparece la clasificación cruzada 
de sexo, edad y estado civil - en que se situaba aproximadamente en los 24 años, hasta llegar a los 26,7 en 1940 para las mujeres y superar los 29 para los varones. Es a partir de 1950 cuando empieza a reducirse de manera sostenida la edad media al matrimonio, y la proporción final de casadas aumenta progresivamente hasta alcanzar el $91 \%$ con las cohortes nacidas en torno a los años de la Guerra Civil. Son las últimas que registran proporciones finales de esa envergadura, pues entre las siguientes generaciones, la proporción final de casadas ha descendido ininterrumpidamente. Los efectos de esta reducción en la intensidad de la nupcialidad se pueden observar en la proporción de casadas que se aprecia en los sucesivos censos (Delgado, 1993), los cuales muestran una continua reducción, perceptible en todos los grupos de edad.

Entre 1950 y 1980 se reduce la edad a las primeras nupcias entre las españolas, para luego volver a incrementarse de manera ininterumpida hasta el presente. Tanto el descenso iniciado a mediados de siglo como el posterior aumento son rasgos que se aprecian en el resto de los países europeos, aunque más tempranamente. Según puede observarse en el Cuadro I, entre 1970 y 1975 los países nórdicos, junto con Francia y el Reino Unido, ya presentaban incrementos en la edad media a la primera nupcialidad, mientras que todo el sur -España, Grecia, Italia y Portugal- todavía se encontraban en la fase anterior de rejuvenecimiento del calendario de la nupcialidad. No eran los únicos, pues también algunos otros acusaban descensos aunque, en general, de menor entidad. Entre 1975 y 1980, la mayor parte de los países habían incorporado la nueva pauta de un matrimonio tardío y sólo seguía disminuyendo la edad al casarse en Grecia, Portugal y España, junto con Irlanda y Luxemburgo. A excepción de Italia, que muestra algo más de precocidad, es después de 1980 cuando el sur se incorpora a las nuevas tendencias de retraso del matrimonio.

Esta evolución conduce a que en 1995 son Suecia y Dinamarca los países en los que las mujeres presentan una edad media más elevada al contraer el primer matrimonio - en torno a los 29 años-, mientras que la edad más joven es la de Grecia. España, que, junto con Irlanda, tenía en 1970 el calendario más retrasado, muestra una posición intermedia en 1995. Lo que se aprecia al observar las cifras es un retraso por parte de los países del sur en adoptar las nuevas tendencias anticipadas por los nórdicos. 
En la segunda parte del Cuadro I puede apreciarse el índice sintético de primonupcialidad femenina, que es una medida resumen de la proproción final de casadas que alcanzaría esa cohorte ficticia que son el conjunto de generaciones en un año dado.

La edad media al primer matrimonio, al igual que el índice sintético de primonupcialidad no refleja la misma realidad social en los países del sur que en los del norte. En éstos, el matrimonio con frecuencia va precedido de un período de cohabitación y, además, muchas de las parejas que cohabitan no llegan a casarse posteriormente. Cosa diferente ocurre en los países del sur de Europa, donde la cohabitación es un fe-

\section{CuAdro I}

EDAD MEDIA AL PRIMER MATRIMONIO E ÍNDICE SINTÉTICO DE PRIMERA NUPCIALIDAD (MUJERES) EN LA EUROPA DE LOS QUINCE, 1970-1995

A) Edad media al primer matrimonio

\begin{tabular}{lllllll}
\hline & $\mathbf{1 9 7 0}$ & $\mathbf{1 9 7 5}$ & $\mathbf{1 9 8 0}$ & $\mathbf{1 9 8 5}$ & $\mathbf{1 9 9 0}$ & $\mathbf{1 9 9 5}$ \\
\hline Alemania & & & & & 25,5 & 27,3 \\
$\quad$ RFA & 23,0 & 22,7 & 23,4 & 24,6 & 25,9 & 27,5 \\
$\quad$ RDA & 21,9 & 21,8 & 21,8 & 22,7 & 23,7 & 26,4 \\
Austria & 23,1 & 22,8 & 23,1 & 24,0 & 25,1 & 26,7 \\
Bélgica & 22,4 & 21,6 & 22,3 & 23,3 & 24,5 & 25,8 \\
Dinamarca & 22,8 & 23,7 & 24,8 & 26,3 & 27,6 & 29,1 \\
Finlandia & 23,0 & 23,5 & 24,5 & 25,4 & 26,5 & 27,6 \\
Francia & 22,4 & 22,5 & 23,0 & 24,2 & 25,6 & 26,9 \\
Grecia & 22,9 & 22,6 & 22,3 & 22,8 & 23,8 & 25,3 \\
Holanda & 22,7 & 22,6 & 23,1 & 24,4 & 25,9 & 27,4 \\
Irlanda & 24,8 & 24,4 & 24,1 & 25,0 & 26,3 & 27,8 \\
Italia & 24,1 & 23,5 & 24,1 & 24,5 & 25,6 & 26,9 \\
Luxemburgo & 23,2 & 23,3 & 23,0 & 24,1 & 25,4 & 26,8 \\
Portugal & 24,3 & 23,7 & 23,3 & 23,6 & 24,2 & 24,9 \\
Reino Unido & 22,4 & 22,8 & 23,0 & 23,8 & 25,2 & 26,7 \\
Suecia & 24,0 & 25,1 & 26,4 & 27,5 & 27,6 & 28,7 \\
España & 24,8 & 24,2 & 23,7 & 24,6 & 25,5 & 27,0 \\
\hline
\end{tabular}


CuAdro I (Continuación)

B) Índice sintético de primera nupcialidad

\begin{tabular}{lcccccc}
\hline & $\mathbf{1 9 7 0}$ & $\mathbf{1 9 7 5}$ & $\mathbf{1 9 8 0}$ & $\mathbf{1 9 8 5}$ & $\mathbf{1 9 9 0}$ & $\mathbf{1 9 9 5}$ \\
\hline Alemania & & & & & & 0,57 \\
$\quad$ RFA & 0,97 & 0,76 & 0,66 & 0,60 & 0,64 & 0,60 \\
$\quad$ RDA & 0,98 & 0,92 & 0,81 & 0,74 & 0,64 & 0,40 \\
Austria & 0,91 & 0,75 & 0,68 & 0,60 & 0,58 & 0,55 \\
Bélgica & 0,99 & 0,89 & 0,77 & 0,66 & 0,72 & 0,57 \\
Dinamarca & & 0,66 & 0,53 & 0,57 & 0,60 & 0,66 \\
Finlandia & 0,94 & 0,71 & 0,67 & 0,58 & 0,58 & 0,57 \\
Francia & 0,92 & 0,86 & 0,71 & 0,54 & 0,56 & 0,49 \\
Grecia & 1,06 & 1,16 & 0,87 & 0,83 & 0,72 & 0,75 \\
Holanda & 1,06 & 0,83 & 0,68 & 0,57 & 0,66 & 0,53 \\
Irlanda & & 0,93 & 0,83 & 0,68 & 0,69 & 0,59 \\
Italia & 1,00 & 0,93 & 0,77 & 0,68 & 0,69 & 0,62 \\
Luxemburgo & 0,89 & 0,80 & 0,66 & 0,57 & 0,65 & 0,56 \\
Portugal & 1,09 & 1,27 & 0,81 & 0,78 & 0,84 & 0,77 \\
Reino Unido $*$ & 1,04 & 0,88 & 0,77 & 0,67 & 0,62 & 0,53 \\
Suecia & 0,62 & 0,63 & 0,53 & 0,55 & 0,55 & 0,44 \\
España & 1,01 & 1,04 & 0,78 & 0,65 & 0,69 & 0,60 \\
\hline
\end{tabular}

* Datos referidos sólo a Inglaterra y Gales.

Fuente: Council of Europe (1998). Para España: 1975 y 1995 elaboración propia.

nómeno minoritario y donde, con frecuencia, es un paso previo al matrimonio y no una situación definitiva.

\section{EVOLUCIÓN RECIENTE DE LA NUPCIALIDAD EN ESPAÑA}

El indice sintético de primera nupcialidad y las tasas específicas por edad

En 1975 el índice sintético de nupcialidad superó la unidad en el conjunto de España $(1,040)$ como resultado del adelanto que se estaba produciendo en la edad al contraer matrimonio. Sólo seis comunidades 
registraron valores inferiores a uno, resultando el menor el 0,838 de Extremadura, mientras que la intensidad más elevada había sido la de Baleares, que acusaba 1,214 (Cuadro II).

CUADRO II

ÍNDICE SINTÉTICO DE PRIMERA NUPCIALIDAD DE MUJERES. ESPAÑA, 1975-1995

\begin{tabular}{lccccc}
\hline CC.AA. & $\mathbf{1 9 7 5}$ & $\mathbf{1 9 8 1}$ & $\mathbf{1 9 8 6}$ & $\mathbf{1 9 9 1}$ & $\mathbf{1 9 9 5}$ \\
\hline Andalucía & 1,010 & 0,768 & 0,709 & 0,711 & 0,622 \\
Aragón & 1,050 & 0,690 & 0,677 & 0,684 & 0,598 \\
Asturias & 0,995 & 0,686 & 0,684 & 0,648 & 0,532 \\
Baleares & 1,214 & 0,731 & 0,743 & 0,726 & 0,640 \\
Canarias & 0,947 & 0,732 & 0,640 & 0,625 & 0,543 \\
Cantabria & 1,001 & 0,720 & 0,611 & 0,611 & 0,537 \\
Castilla-La Mancha & 0,885 & 0,765 & 0,713 & 0,718 & 0,655 \\
Castilla y León & 0,893 & 0,524 & 0,570 & 0,610 & 0,540 \\
Cataluña & 1,080 & 0,604 & 0,642 & 0,683 & 0,611 \\
C.Valencia & 1,114 & 0,767 & 0,638 & 0,661 & 0,622 \\
Extremadura & 0,838 & 0,789 & 0,728 & 0,720 & 0,620 \\
Galicia & 0,952 & 0,666 & 0,618 & 0,632 & 0,544 \\
Madrid & 1,171 & 0,680 & 0,657 & 0,681 & 0,594 \\
Murcia & 1,034 & 0,766 & 0,756 & 0,710 & 0,645 \\
Navarra & 1,052 & 0,687 & 0,613 & 0,633 & 0,615 \\
País Vasco & 1,091 & 0,632 & 0,555 & 0,559 & 0,529 \\
Rioja & 1,042 & 0,667 & 0,682 & 0,662 & 0,587 \\
\multicolumn{1}{c}{ Total Nacional * } & $\mathbf{1 , 0 4 0}$ & $\mathbf{0 , 7 7 9 *}$ & $\mathbf{0 , 6 6 1}$ & $\mathbf{0 , 6 7 5}$ & $\mathbf{0 , 6 0 2}$ \\
\hline
\end{tabular}

* Corregido el subregistro de matrimonios para el Total Nacional en 1981.

Fuente: I.N.E., Secretariado General de la Conferencia Episcopal y elaboración propia.

Como se aprecia en el Gráfico I, desde 1975 hasta 1986 el índice sintético muestra una reducción, que se cifra para el período en un 36\%. Entre 1986 y 1991, se produce una leve recuperación del $2 \%$, para proseguir de nuevo un descenso que continúa hasta 1995, momento en que el índice alcanza el 0,602, un valor 42\% menor que en 1975 . 


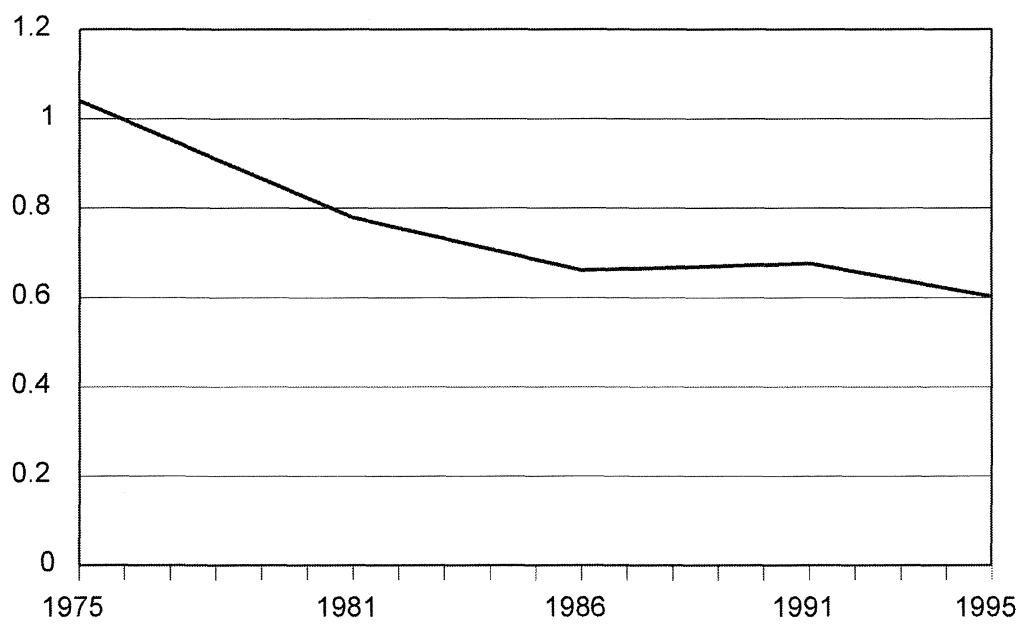

Fuente: Cuadro II.

GRÁfICO I.-Índice de primera nupcialidad de mujeres. España, 1975-1995

Basándose solamente en los valores que toma el índice sintético de primera nupcialidad femenina, se podría pensar que la nupcialidad en España ha descendido de manera generalizada y que la tímida fluctuación producida entre 1986 y 1991 fue un breve episodio de cambio de calendario. Las tasas específicas de nupcialidad por edad que se muestran en el Cuadro III, permiten apreciar que los cambios fueron de diferente magnitud para los distintos grupos de edad según el período y no siempre siguieron la misma dirección. Entre 1975 y 1981 las tasas descendieron en torno al 20-30\% en los grupos de 20-40 años, mientras que en el de más edad la reducción se situó entre el 35-40\%. Los datos muestran que en este período el descenso fue generalizado, debido en parte a los altos niveles de nupcialidad de principios de los setenta. Las mujeres de 15-19 años fueron el grupo que experimentó una reducción más moderada, pues sus tasas en 1981 disminuyeron un 7\% en relación a las mujeres de este mismo grupo de edad en 1975.

Entre 1981 y 1995 el descenso se concentra entre las menores de veinticinco años — además, de manera acusada—, pero en el resto, las tasas muestran aumentos en casi todos los casos, excepto las mayores 


\section{CuAdro III}

TASAS ESPECÍFICAS E ÍNDICE SINTÉTICO DE PRIMERA NUPCIALIDAD DE MUJERES. ESPAÑA, 1975-1995

Primeros matrimonios/Total mujeres

\begin{tabular}{lrcccc}
\hline Edad & $\mathbf{1 9 7 5}$ & $\mathbf{1 9 8 1} *$ & $\mathbf{1 9 8 6}$ & $\mathbf{1 9 9 1}$ & $\mathbf{1 9 9 5}$ \\
\hline $15-19$ & 30,05 & 27,89 & 17,75 & 11,02 & 6,33 \\
$20-24$ & 116,13 & 88,01 & 64,55 & 54,11 & 36,21 \\
$25-29$ & 44,32 & 31,97 & 37,80 & 52,11 & 54,20 \\
$30-34$ & 10,01 & 7,13 & 8,12 & 12,82 & 17,42 \\
$35-39$ & 3,56 & 2,78 & 2,59 & 3,52 & 4,37 \\
$40-44$ & 2,05 & 1,30 & 1,21 & 1,32 & 1,52 \\
45.49 & 1,35 & 0,84 & 0,70 & 0,76 & 0,73 \\
I.S.N.** & 1,040 & 0,779 & 0,661 & 0,675 & 0,602 \\
\hline
\end{tabular}

* Corregido el subregistro de matrimonios.

** Calculado por edades simples.

I.S.N. = Índice Sintético de Nupcialidad.

Fuente: I.N.E., Secretariado General de la Conferencia Episcopal y elaboración propia.

de treinta y cinco años entre 1981-86 y las mayores de cuarenta y cinco entre 1991-95.

Considerando en su conjunto los últimos veinte años de historia de la nupcialidad española, se puede afirmar que, aunque el índice sintético de primera nupcialidad femenina ha descendido un $42 \%$, las tasas de nupcialidad de las mujeres entre 25-39 años son más elevadas en 1995 que en 1975 y sólo el resto de los grupos muestra tasas menores. Así, la reducción de la intensidad total de la nupcialidad se debe al gran peso que tienen los grupos de 15-24 años, cuyas tasas han experimentado descensos alrededor del 70-80\%.

$\mathrm{Al}$ estar calculado el índice tomando en el numerador los primeros matrimonios por edad de la contrayente y en el denominador las mujeres de esa edad, conforme las mujeres se van casando, quedan menos de ellas «en riesgo» de contraer su primer matrimonio, por lo que la tasa, en períodos posteriores a aquellos de alta nupcialidad, ha de acusar necesariamente cierto descenso, parte del cual es artificial y derivado de la 
propia metodología de cálculo, como ya han señalado otros autores (Miret, 1994).

Para eliminar este efecto de estructura por estado civil en el cálculo de las tasas, se han recalculado éstas tomando en el numerador los primeros matrimonios y en el denominador las mujeres solteras o «en riesgo» de contraer un primer matrimonio. Los datos obtenidos por este procedimiento se muestran en el Cuadro IV.

\section{CuAdro IV}

TASAS ESPECÍFICAS E ÍNDICE SINTÉTICO DE PRIMERA NUPCIALIDAD DE MUJERES. ESPAÑA, 1975-1995

Primeros matrimonios/Mujeres solteras

\begin{tabular}{lrrrrr}
\hline Edad & $\mathbf{1 9 7 5}$ & $\mathbf{1 9 8 1}^{*}$ & $\mathbf{1 9 8 6}$ & $\mathbf{1 9 8 1}$ & $\mathbf{1 9 9 5}$ \\
\hline $15-19$ & 31,21 & 29,72 & 18,43 & 11,25 & 6,42 \\
$20-24$ & 185,30 & 149,68 & 91,63 & 69,52 & 40,84 \\
$25-29$ & 189,93 & 145,06 & 127,53 & 134,85 & 104,14 \\
$30-34$ & 77,85 & 57,47 & 56,34 & 74,30 & 83,99 \\
$35-39$ & 36,28 & 28,71 & 24,73 & 31,65 & 41,17 \\
$40-44$ & 21,42 & 14,47 & 13,48 & 14,75 & 17,11 \\
45.49 & 12,47 & 8,97 & 8,12 & 9,32 & 10,97 \\
\hline
\end{tabular}

* Corregido el subregistro de matrimonios.

Fuente: I.N.E., Secretariado General de la Conferencia Episcopal y elaboración propia.

Comparando el Cuadro IV con el III, se observa que en el grupo de edad más joven ambas tasas resultan lógicamente similares, pero en los restantes difieren sensiblemente, por los motivos ya explicados anteriormente acerca del efecto de la nupcialidad anterior. De acuerdo a las nuevas tasas recalculadas, en los dos primeros subperíodos (1975-1981 y 1981-1986) todos los grupos de edad reducen su tasa de nupcialidad, es decir, la propensión a contraer matrimonio es menor entre las solteras de los años ochenta de lo que lo era entre las de los setenta. Entre 1986 y 1991 desciende la tasa de primeros matrimonios entre las mujeres solteras menores de veinticinco años, pero aumenta la tasa en el res- 
to de los grupos respecto al período anterior, consecuencia en parte de una recuperación de los matrimonios no celebrados anteriormente. Esta tendencia se mantiene, aunque más débilmente en los años noventa, pues sólo se observa una ligera recuperación de la tasa entre las que sobrepasan la treintena.

Considerando los veinte años transcurridos a través de un indicador neto de primera nupcialidad - como son las tasas calculadas sobre la población que permanece soltera- se aprecia de manera más nítida que el descenso en la intensidad de la nupcialidad que muestra el índice sintético es real, pero que también se ha producido un cambio en las pautas por edad respecto a la propensión a contraer matrimonio. En los setenta y ochenta, las mujeres que no se casaban a edades relativamente tempranas, tenían menor propensión a casarse una vez superados los treinta años, mientras que en 1995, acceden al matrimonio en mayor medida, pues las tasas entre solteras de 30-39 años son más altas de lo que lo eran en cualquier momento anterior, incluido 1975, momento de máxima intensidad de la nupcialidad. En los Gráficos II y III se pueden apreciar estas variaciones, que revelan las importantes transformaciones del calendario, así como la reducción de la propensión a contraer matrimonio, generalizada entre las menores de 25 años, y casi generalizada entre las de 25-29, pues en este grupo sólo se aprecia la excepción del año 1991.

Es de destacar cómo la diferente metodología de cálculo puede hacer variar la interpretación: si se observa el gráfico que muestra las tasas calculadas tomando en el denominador el conjunto de mujeres, se observa que la tasa más elevada es la que registra el grupo de 20-24 años en todas las fechas consideradas, excepto en 1995, en que la tasa más alta corresponde al grupo de 25-29, si bien en 1991 ambos grupos ya se aproximaban en sus valores. Es decir, con el indicador menos refinado, los cambios en el calendario no se dejan ver nítidamente hasta que el retraso es muy pronunciado, como ocurre en los noventa. Por el contrario, con el indicador neto - tasas específicas sobre la población soltera-, en primer lugar se aprecia que las tasas más elevadas corresponden siempre al grupo de 25-29 años y no al de 20-24, como ocurría con el indicador anterior, con la única excepción del año 1981. Así pues, una vez que se elimina el efecto de la nupcialidad previa en cada grupo de edad, el de 25-29 años aparece como el que muestra una propensión más elevada a contraer matrimonio. 


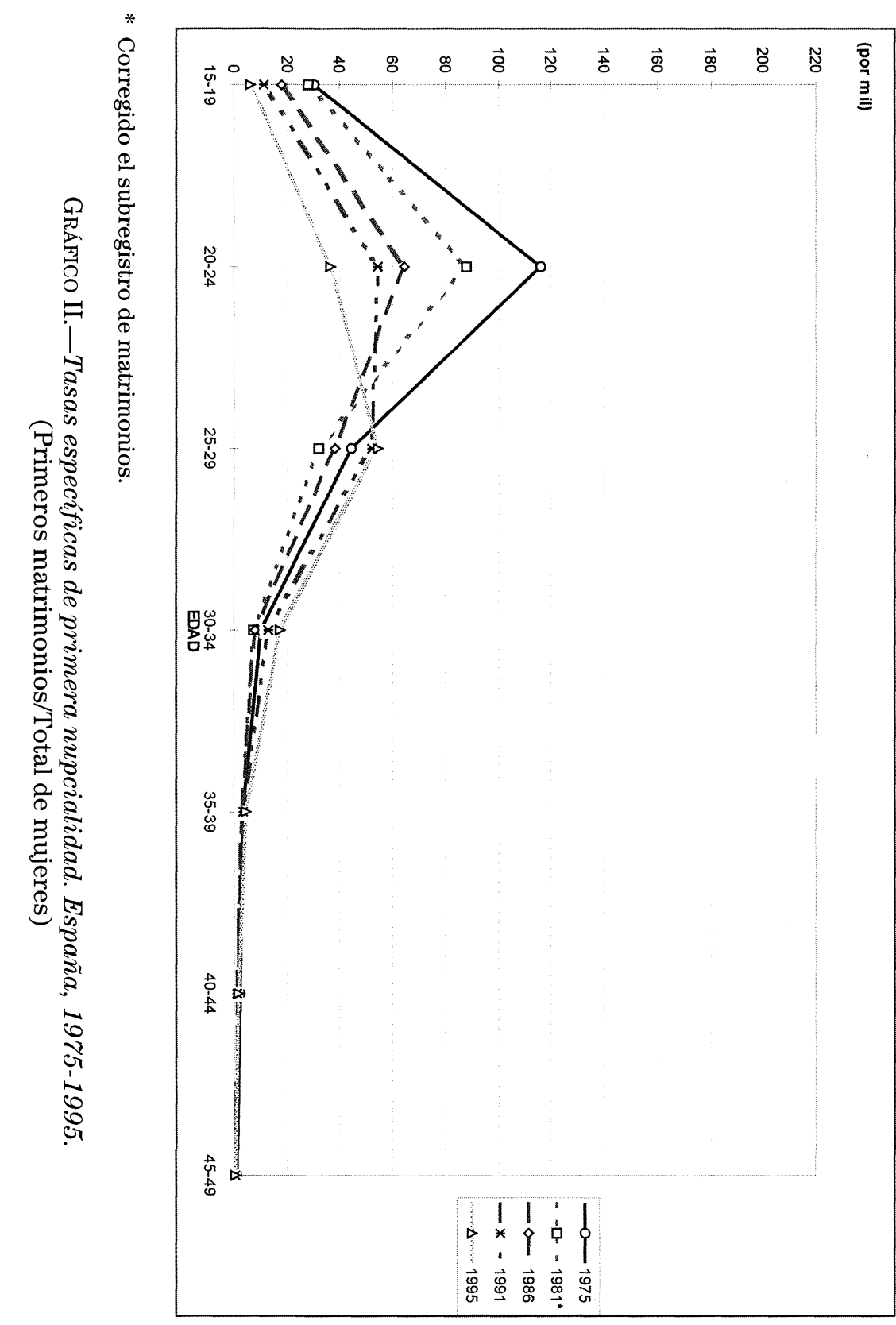

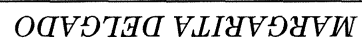




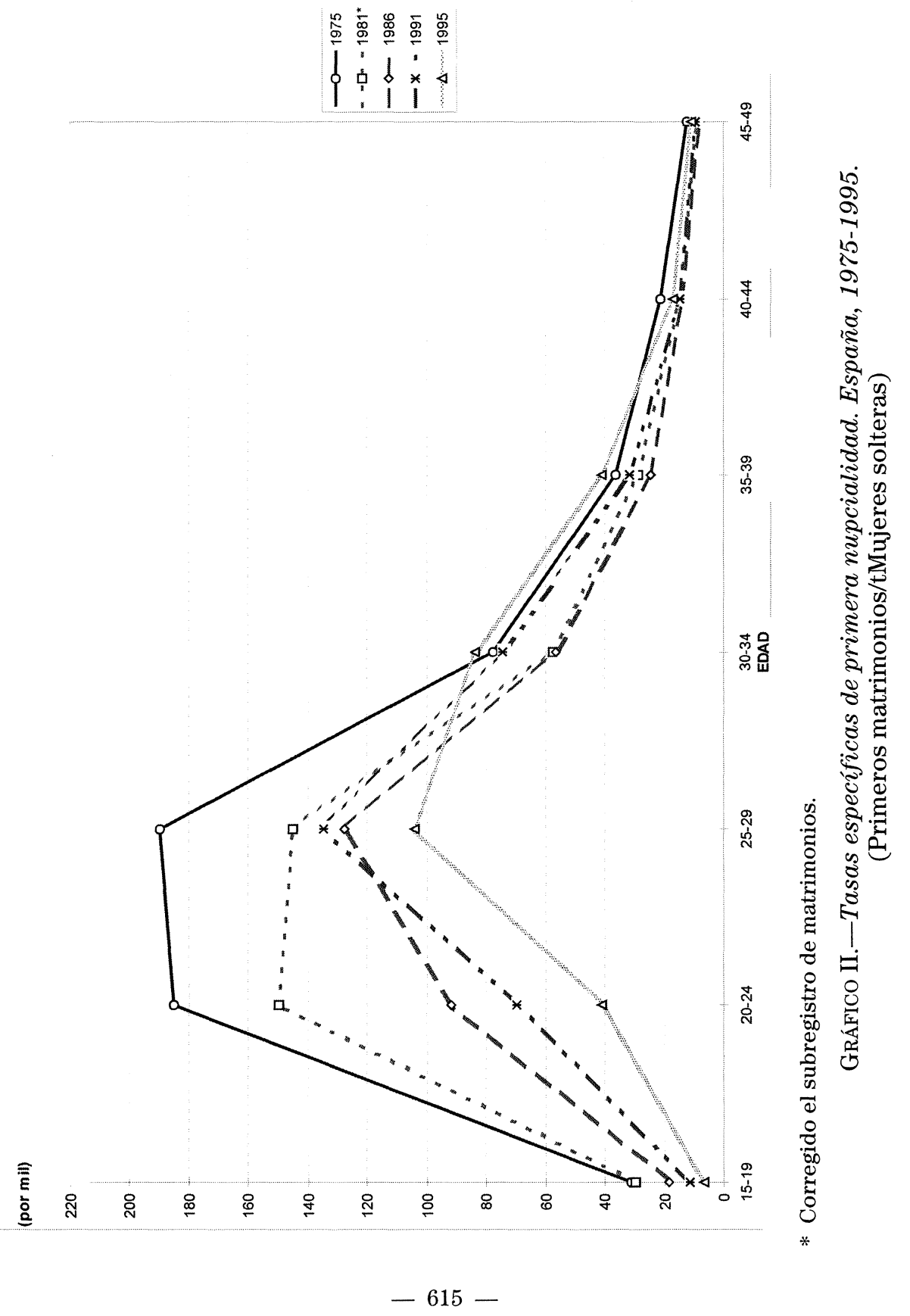


La edad media al primer matrimonio

La edad media a la primera nupcialidad de las mujeres constituye una medida resumen que ratifica lo observado a través de las tasas en cuanto a variaciones del calendario se refiere. Calculada a través de las estadísticas vitales, en 1975 se aprecia que la edad media a la que contraían matrimonio las mujeres estaba un poco por debajo de los veinticuatro años (Cuadro V). Entre esta fecha y 1981 descendió ligeramente, para mostrar a continuación una tendencia al incremento, que no se ha detenido todavía, pues a tenor de los datos disponibles la edad promedio al primer matrimonio de las españolas se sitúa casi en 27

CUADRO V

EDAD MEDIA AL PRIMER MATRIMONIO (MUJERES). ESPAÑA, 1975-1995

\begin{tabular}{lcccccc}
\hline CC.AA. & $\mathbf{1 9 7 5}$ & $\mathbf{1 9 8 1}$ & $\mathbf{1 9 8 6}$ & $\mathbf{1 9 9 1}$ & $\mathbf{1 9 9 5}$ & $\begin{array}{c}\text { Cambio } \\
\mathbf{1 9 7 5 - 1 9 9 5}\end{array}$ \\
\hline Andalucía & 23,83 & 23,34 & 24,08 & 25,09 & 26,27 & 2,44 \\
Aragón & 24,63 & 24,40 & 25,15 & 26,32 & 27,50 & 2,87 \\
Asturias & 23,39 & 23,43 & 24,36 & 25,57 & 26,99 & 3,60 \\
Baleares & 23,38 & 23,28 & 24,01 & 25,25 & 26,44 & 3,06 \\
Canarias & 22,91 & 22,67 & 23,56 & 24,97 & 26,12 & 3,21 \\
Cantabria & 23,70 & 23,65 & 24,66 & 25,79 & 27,44 & 3,74 \\
Castilla-La Mancha & 24,35 & 23,95 & 24,44 & 25,35 & 26,25 & 1,90 \\
Castilla y León & 24,80 & 24,45 & 25,14 & 26,36 & 27,75 & 2,95 \\
Cataluña & 23,44 & 23,14 & 24,31 & 25,69 & 26,80 & 3,36 \\
C.Valencia & 23,87 & 23,35 & 24,32 & 25,38 & 26,52 & 2,65 \\
Extremadura & 24,23 & 23,82 & 23,97 & 24,99 & 26,15 & 1,92 \\
Galicia & 23,31 & 23,16 & 23,65 & 24,78 & 26,08 & 2,77 \\
Madrid & 24,41 & 24,32 & 25,56 & 26,75 & 28,02 & 3,61 \\
Murcia & 23,13 & 23,01 & 23,82 & 24,79 & 25,87 & 2,74 \\
Navarra & 25,17 & 24,85 & 25,63 & 26,70 & 27,87 & 2,70 \\
País Vasco & 24,33 & 24,29 & 25,52 & 27,19 & 28,35 & 4,02 \\
Rioja & 24,55 & 24,02 & 24,58 & 25,98 & 27,34 & 2,79 \\
$\quad$ Total Nacional * & $\mathbf{2 3 , 9 2}$ & $\mathbf{2 3 , 6 0}$ & $\mathbf{2 4 , 4 8}$ & $\mathbf{2 5 , 6 7}$ & $\mathbf{2 6 , 8 6}$ & $\mathbf{2 , 9 4}$ \\
\hline
\end{tabular}

Fuente: I.N.E., Movimiento Natural de la Población y elaboración propia. 

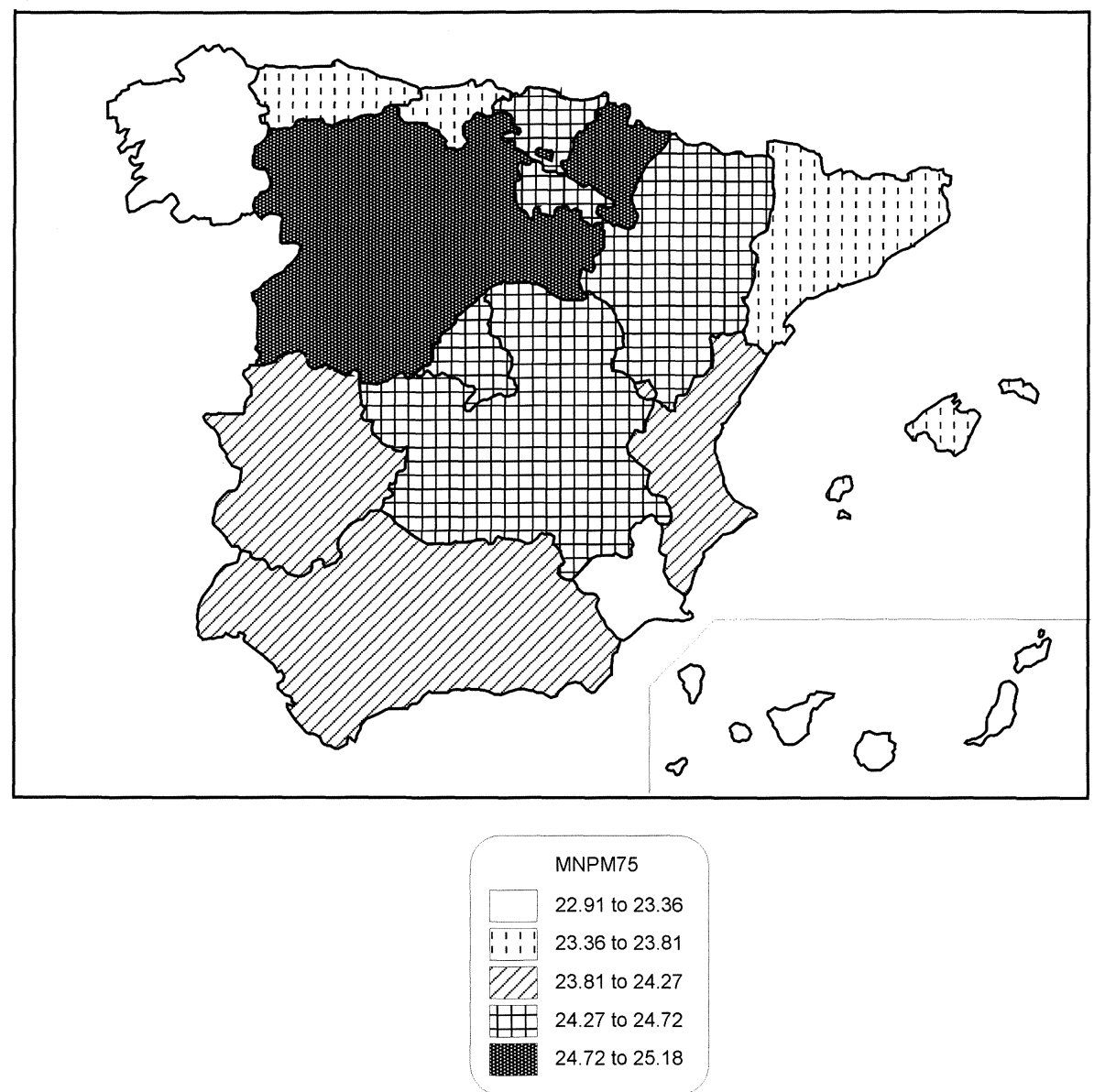

MAPA 1.-Edad media al primer matrimonio mujeres (MNP), 1975.

años en 1995, lo que significa un aumento de tres años en el transcurso de las dos décadas analizadas.

Esta tendencia se manifiesta prácticamente en todas las comunidades autónomas. Aunque el incremento en la edad media a la que las mujeres contraen las primeras nupcias para el conjunto de España está en torno a los 3 años, se aprecian comunidades donde se ha retrasado la 


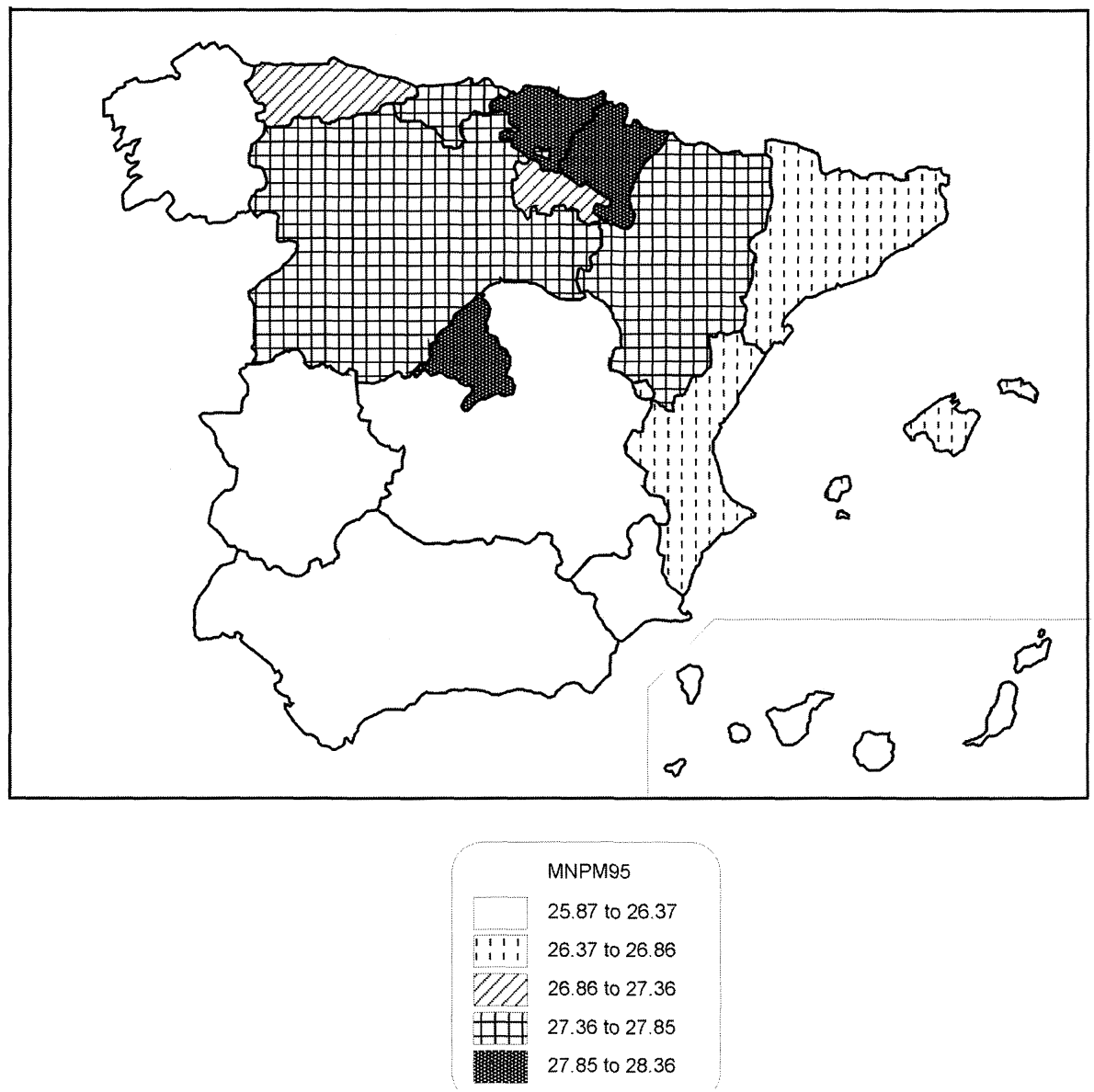

MAPA 2.-Edad media al primer matrimonio mujeres (MNP), 1995.

edad al matrimonio más de 3,5 años, como es el caso de Asturias, Cantabria y Madrid. Más llamativo aún resulta el País Vasco, donde el retraso ha sido de 4 años. Por el contrario, las comunidades donde menos se ha retrasado el calendario de la nupcialidad, con valores inferiores a 2 años, son Castilla-La Mancha y Extremadura.

En los Mapas 1 y 2 se puede apreciar la situación de las distintas comunidades autónomas en 1975 y 1995 respectivamente, en cuanto a la 
edad de las mujeres a su primer casamiento. En la primera fecha, Galicia, Murcia y Canarias eran las zonas de matrimonio más precoz, seguidas de Asturias, Baleares, Cantabria y Cataluña, mientras que Castilla y León, además de Navarra y buena parte del interior, presentaban un matrimonio más tardío.

En 1995 persisten como zonas de nupcialidad temprana en el contexto español Canarias, Galicia y Murcia, pero se han incorporado al grupo Andalucía, Castilla-La Mancha y Extremadura, comunidades donde la edad al matrimonio ha experimentado un incremento sólo moderado. No deja de ser significativo que las comunidades con un patrón de matrimonio más temprano en 1995 son, con la excepción de Galicia, las zonas del país con más alta fecundidad, lo que refleja la influencia que tienen en España las pautas de nupcialidad en los índices de reproducción.

\section{CuAdro VI}

EDAD MEDIA EL PRIMER MATRIMONIO (SMAM), 1975-1991

A) Mujeres

\begin{tabular}{lllll}
\hline CC.AA. & $\mathbf{1 9 7 5}$ & $\mathbf{1 9 8 1}$ & $\mathbf{1 9 8 6}$ & $\mathbf{1 9 9 1}$ \\
\hline Andalucía & 23,38 & 23,07 & 24,46 & 25,47 \\
Aragón & 24,07 & 24,01 & 25,21 & 26,47 \\
Asturias & 22,85 & 23,08 & 24,64 & 26,22 \\
Baleares & 22,14 & 22,34 & 23,99 & 25,33 \\
Canarias & 21,68 & 22,00 & 23,84 & 25,36 \\
Cantabria & 23,13 & 23,19 & 24,78 & 26,37 \\
Castilla-La Mancha & 24,66 & 24,55 & 25,13 & 25,88 \\
Castilla y León & 24,94 & 24,56 & 25,67 & 26,90 \\
Cataluña & 22,61 & 22,33 & 24,44 & 26,08 \\
C.Valencia & 23,03 & 22,86 & 24,36 & 25,76 \\
Extremadura & 25,00 & 24,13 & 24,93 & 25,58 \\
Galicia & 22,48 & 22,00 & 23,40 & 25,04 \\
Madrid & 23,29 & 23,55 & 25,81 & 27,15 \\
Murcia & 22,61 & 22,64 & 24,19 & 25,23 \\
Navarra & 23,98 & 24,11 & 25,56 & 27,03 \\
País Vasco & 22,85 & 23,55 & 25,95 & 27,87 \\
Rioja & 23,89 & 23,76 & 24,84 & 26,03 \\
\multicolumn{1}{c}{ Total Nacional $*$} & $\mathbf{2 3 , 2 3}$ & $\mathbf{2 3 , 1 1}$ & $\mathbf{2 4 , 7 5}$ & $\mathbf{2 6 , 0 9}$ \\
\hline
\end{tabular}


B) Varones

\begin{tabular}{lllll}
\hline CC.AA. & $\mathbf{1 9 7 5}$ & $\mathbf{1 9 8 1}$ & $\mathbf{1 9 8 6}$ & $\mathbf{1 9 9 1}$ \\
\hline Andalucía & 26,74 & 25,79 & 26,80 & 27,77 \\
Aragón & 25,58 & 26,58 & 27,39 & 28,61 \\
Asturias & 26,41 & 25,73 & 27,03 & 28,53 \\
Baleares & 26,18 & 25,54 & 26,60 & 27,75 \\
Canarias & 26,22 & 25,68 & 26,93 & 28,17 \\
Cantabria & 26,95 & 25,78 & 27,11 & 28,67 \\
Castilla-La Mancha & 27,55 & 26,81 & 27,39 & 28,22 \\
Castilla y León & 28,63 & 27,86 & 28,55 & 29,45 \\
Cataluña & 26,29 & 25,33 & 26,99 & 28,42 \\
C.Valencia & 26,35 & 25,73 & 26,85 & 28,04 \\
Extremadura & 27,87 & 26,78 & 27,25 & 27,83 \\
Galicia & 26,83 & 26,08 & 26,97 & 27,99 \\
Madrid & 26,29 & 26,05 & 27,99 & 29,27 \\
Murcia & 26,22 & 25,59 & 26,73 & 27,60 \\
Navarra & 27,62 & 27,02 & 28,10 & 29,42 \\
País Vasco & 26,38 & 26,38 & 28,23 & 30,06 \\
Rioja & 27,28 & 26,49 & 27,29 & 28,26 \\
\multicolumn{1}{c}{ Total Nacional $*$} & $\mathbf{2 6 , 7 3}$ & $\mathbf{2 6 , 0 1}$ & $\mathbf{2 7 , 2 7}$ & $\mathbf{2 8 , 4 6}$ \\
\hline
\end{tabular}

Fuente: I.N.E., Censos de 1981 y 1991, Padrones de 1975 y 1986 y elaboración propia.

Si se compara la evolución entre las fechas para las que se dispone de la edad media al primer matrimonio calculada a partir de distintas fuentes y con diferente metodología - estadísticas vitales (Cuadro V) y fuentes censales, mediante la SMAM (Cuadro VI)—, se aprecia que la tendencia es idéntica en el Total Nacional, pues se observa con cualquiera de los dos procedimientos de cálculo un ligero rejuvenecimiento en la edad media al primer matrimonio entre 1975 y 1981, al que sigue un posterior retraso en todos los períodos observados. La cuantía del descenso y del posterior aumento es diferente debido a la metodología empleada, ya que la elaboración del indicador a través de los censos recoge la historia nupcial de un conjunto de generaciones, pero al calcularse a través de las proporciones de soltería de los grupos, y tener todos ellos el mismo peso en el resultado final — se multiplica por cinco la pro- 
porción de solteros en el grupo quinquenal-, ante un aumento de las proporciones en los grupos más jóvenes, se eleva la edad resultante ${ }^{8}$. Sin embargo, la edad media que se calcula a partir del M.N.P. está ponderada por las tasas de nupcialidad por edades simples. El retraso que se aprecia en la edad al matrimonio entre 1975 y 1991 resulta de mayor cuantía utilizando los datos de los recuentos de población.

En relación a las comunidades autónomas, la tendencia que se observa con uno u otro indicador es la misma en la mayor parte de los casos. Cabe hacer la excepción de que el rejuvenecimiento - leve, por otra parte- que se constata entre 1975 y 1981 en la edad de las mujeres no es tan general utilizando la SMAM como con los datos del M.N.P., mientras que el posterior retraso adquiere características de casi universal con cualquiera de los dos indicadores.

La diferencia entre la edad media de los varones y mujeres al primer matrimonio era en 1975 de 3,5 años para el conjunto del país, oscilando entre 1,5 en Aragón y 4,5 en Canarias. La distancia fue reduciéndose hasta 1991, en que el conjunto del país acusa una diferencia entre hombres y mujeres a la hora de matrimoniar en torno a los 2,4 años. La pauta de acercamiento entre ambos colectivos en la edad al matrimonio es perceptible en la casi totalidad de las comunidades autónomas, salvo en Aragón, donde la diferencia, en lugar de reducirse, se incrementó en casi un año.

\section{CONCLUSIONES}

En este trabajo se ha abordado el estudio de la nupcialidad a partir de dos fuentes distintas y con diferente enfoque metodológico. Lo que se ha mostrado respecto a la edad media al primer matrimonio es que, cuando varía la fuente, los resultados pueden diferir algo respecto a la cuantía, pero no respecto a la tendencia. Sin embargo, al analizar las tasas de nupcialidad por edad, el método de cálculo se revela importante, pues el indicador que contempla la nupcialidad precedente se torna más clarificador respecto a las variaciones que se aprecian en el calendario y respecto a qué grupos corresponde la mayor intensidad. Asimismo, permite apreciar más nítidamente la evolución del período ana-

${ }^{8}$ Para una discusión detallada del método de cálculo y fórmulas, véase Hajnal (1953), así como Newell (1988). 
lizado, que, de otra forma, queda enmascarada en los primeros años del cambio de tendencia.

La observación de las pautas de la nupcialidad en Europa han permitido constatar la existencia en España de unos patrones de comportamiento similares a los del resto de los países europeos, aunque se aprecia un claro retraso en la adopción de las nuevas tendencias por parte de la Europa del sur, retraso que resulta incluso algo más acusado en España. Esto hace que, mientras en gran parte de Europa el modelo de matrimonio tardío y no universal se fue debilitando desde las primeras décadas del siglo xx, en España todavía se mantuvo vigente hasta después de la Guerra Civil. Asimismo, el rejuvenecimiento en el calendario de la nupcialidad que tuvo lugar a comienzos de la segunda mitad del siglo en buena parte de Europa, no se aprecia en España hasta los años sesenta. Del mismo modo que el posterior retraso no fue perceptible hasta los ochenta, una década más tarde que en los países vecinos. No obstante, actualmente España registra la edad al primer matrimonio más elevada entre los países del sur del Continente, y relativamente elevada entre el resto, ya que sólo registran cifras superiores los países nórdicos.

Así, se aprecia que, a mediados de los noventa, la edad media al matrimonio de las mujeres $(26,9)$ alcanza valores incluso superiores a los registrados en $1940(26,6)$ o $1950(26,4)$. Si bien el contexto económico y social, así como los factores subyacentes, son muy distintos, coinciden en un punto: las dificultades ante el matrimonio. Las teorías tradicionales que tratan de explicar las pautas de nupcialidad tardía en Europa Occidental, destacan la relación entre la edad al matrimonio y la edad a la que se alcanza la independencia económica. La constitución de una familia - ya sea por la vía del matrimonio tradicional o mediante una unión consensual - necesita una base material que la sustente (Watkins, 1981). Eso explicaría por qué en España el descenso de la nupcialidad no se ha visto contrapesado por un aumento de las uniones consensuales, las cuales necesitarían, asimismo, una base material para su constitución; base que los jóvenes consideran de muy difícil consecución con las altas tasas de desempleo que padecen, a las que se añade los problemas que presenta el mercado inmobiliario.

Sin embargo, el importante descenso de la nupcialidad observado en los últimos quince años, no parece reflejar un rechazo del matrimonio como institución, pues más bien se trata de esos factores exógenos a las preferencias de los individuos - desempleo y vivienda-, que retrasan 
o impiden el matrimonio. En el caso español puede decirse que, a tenor de las respuestas de los ciudadanos cuando se les interroga sobre estas materias, la familia y el matrimonio se encuentran entre las instituciones mejor valoradas ${ }^{9}$. Algo que no parece una respuesta condicionada por la deseabilidad social, pues los comportamientos no la desmienten: la cohabitación - aunque en alza - es todavía un comportamiento bastante minoritario, mostrando que la fórmula predominante de constitución de la familia continúa siendo el matrimonio. Por otra parte, las tasas de fecundidad no matrimonial, aunque se han incrementado, resultan bastante bajas en comparación con otros países europeos ${ }^{10}$. Así pues, la maternidad/paternidad es una opción que en España se elige preferentemente dentro del matrimonio, por lo que parece que el descenso de la nupcialidad no obedece a una «crisis» o rechazo de la institución matrimonial, sino a la conjunción de una serie de factores que dificultan o retrasan el matrimonio. Entre los que operan en ambos sentidos -retrasar y dificultar - están los ya mencionados: las adversas condiciones del mercado de trabajo y su precariedad, así como la situación del mercado inmobiliario. Entre los que lo retrasan, está el aumento de los años dedicados al estudio y formación.

Los cambios de tendencia que aquí se han mostrado, por lo recientes, no permiten asegurar que sean duraderos, pues bien puede tratarse de un simple aplazamiento del matrimonio, fruto de una coyuntura económica adversa para los que están en edad de formar una familia. No obstante, no puede descartarse un retorno a las pautas del pasado en cuanto a matrimonio tardío y no universal, al igual que ocurre entre nuestros vecinos europeos. Pero si los principales obstáculos son de índole económica, será necesario esperar a que un cambio de coyuntura respecto al mercado de trabajo para los más jóvenes demandantes de empleo, permita verificar la persistencia o la transitoriedad de las tendencias apuntadas.

${ }^{9}$ El Centro de Investigaciones Sociológicas solicita periódicamente a los ciudadanos su valoración acerca de una serie de instituciones, entre ellas la familia y el matrimonio. Las series de respuestas pueden encontrarse en el Banco de Datos del CIS. De las respuestas obtenidas en marzo de 1998 - por citar una de las más recientes - se concluye que el 78\% de los entrevistados consideraba, personalmente, el matrimonio importante: el 39\% muy importante y el otro 39\% bastante importante (CIS, 1999).

${ }_{10}$ La proporción de nacidos de madre no casada en España representaba menos del $11 \%$ de todos los nacimientos en 1995, mientras que en Francia y el Reino Unido sobrepasa el 30\% y en los países nórdicos está en torno al 50\% (Council of Europe, 1997). 


\section{BIBLIOGRAFÍA}

Alberdi, I. (1999): La nueva familia española, Madrid, Ed. Taurus.

CACHINERO SÁNCHEZ, B. (1981): «El modelo europeo de matrimonio: evolución, determinantes y consecuencias», R.E.I.S., 15, pp. 33-58.

CACHINERo SÁNCHEZ, B. (1982): «La evolución de la nupcialidad en España (1887-1975)», R.E.I.S., 20, pp. 81-99.

CASTRO MARTín, T. (1993): «Changing nuptiality patterns in contemporary Spain» GENUS, vol IL, n. ${ }^{\text {os }} 1-2$, pp. 79-95.

CEnTRO DE InvestigaCiones SociológiCas (1999): «Hijos y parejas», Datos de Opinión, 17, Madrid.

Coale, A. J., and Treadway, R. (1986): «A Summary of the Changing Distribution of Overall Fertility, Marital Fertility, and the Proportion Married in the Provinces of Europe», en Coale A. J., and Watkins, S. C. (eds.) The Decline of Fertility in Europe, Princeton.

CouncIL OF EuROPE (1998): Recent demographic developments in Europe, Council of Europe, Strasbourg.

Delgado, M. (1993): «Cambios recientes en el proceso de formación de la familia», R.E.I.S., 64, pp. 123-153.

Delgado, M., y CAStro Martín, T. (1998): Encuesta de Fecundidad y Familia 1995 (FFS), Serie Actitudes y Opiniones, 20, Centro de Investigaciones Sociológicas, Madrid.

Delgado Pérez, M., y Fernández Cordón, J. A. (1989): «Análisis de las cifras de matrimonios en España desde 1975», Estadística Española, vol. 31, 121, pp. 281-295.

FERNÁNDEZ CoRdón, J. A. (1986): "Análisis longitudinal de la fecundidad en España», Actas del Simposio Internacional sobre Tendencias demográficas y planificación económica, Ministerio de Economía y Hacienda, Madrid, pp. 49-75.

- (1997): «Youth residential independence and autonomy: a comparative study», Journal of Family Issues, vol 18, 6, pp. 576-607.

HAJNAL, J. (1953): «Age at Marriage and Proportions Marrying», Population Studies, 7, 2 , pp. 111-136.

(1965): «European marriage patterns in perspective», en Glass, D. V., and EvERSLEY, D. E. C. (eds.): Population in History: Essays in historical demography, London: Edward Arnold, pp. 101-143.

Instituto NACiONAL DE Estadística (1995): Encuesta de Población Activa. Tablas anuales, Madrid.

LIVI BACCI, M. (1968): «Fertility and Nuptiality Changes in Spain from the Late 18th to the Early 20th Century», Population Studies, 22, 1, pp. 83-102 (part I) y 22, 2, pp. 211-234 (part II).

MIRET, P. (1994): «Análisis provincial de la nupcialidad en España», Papers de Demografía, n. ${ }^{\circ} 86$, Centre d'Estudis Demogràfics, Barcelona.

Miret-GamundI, P. (1997): «Nuptiality patterns in Spain in the eighties», GENUS, vol. LIII, n. ${ }^{\text {os }} 3-4$, pp. $183-198$.

MuÑoz PÉREZ, F. (1995): «Procreación y matrimonio en España (1970-1990)», Revista Internacional de Sociología, 11, pp. 197-238.

Newell, C. (1988): Methods and Models in Demography, London: Belhaven Press.

REHER, D.-S., y VALERo LoBo, A. (1995): Fuentes de información demográfica en España, Centro de Investigaciones Sociológicas, Colección Cuadernos Metodológicos, 13, Madrid.

RowLAND, R. (1987): «Mortalidad, movimientos migratorios y edad de acceso al matrimonio en la Península Ibérica», Boletín de la $A D E H$, año V, 3, pp. 41-63.

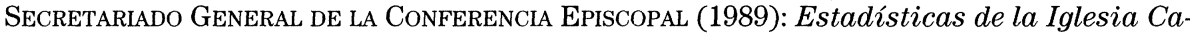
tólica, Madrid. 
WATKINs, S. C. (1981): «Regional patterns of nuptiality in Europe 1870-1960», Population Studies, 35 (2), pp. 199-216.

RESUMEN: En este trabajo se analizan las pautas de nupcialidad de la población española entre 1975 y 1995, haciendo referencia a la evolución histórica y enmarcando la situación de España en el contexto europeo. Mediante indicadores construidos con diferente enfoque metodológico, se aprecia el retraso experimentado por el calendario de la nupcialidad en los años ochenta y noventa, después del rejuvenecimiento en la edad al matrimonio que había tenido lugar en los sesenta y setenta, el cual estuvo acompañado de un aumento de la intensidad. La situación actual representa la vuelta a un modelo de matrimonio tardío y no universal, que ya mostraba España en los años de la posguerra. Asimismo, refleja una evolución análoga a la de otros países europeos, aunque con una cronología algo retrasada.

Palabras Clave: Nupcialidad, España.

ABSTRACT: This study marriage patterns in Spain between 1975 and 1995 views Spanish marriage patterns within the broader European context. A variety of methodologies are used to measure the rise during the 1980s and 1990s in the age at first marriage. This followed a decline in the age at marriage during the 1960s and 1970s with lower rates of permanent celibacy. The marriage pattern of today is characterised by a return to a pattern of late and not universal marriage as present in Spain between the end of the Civil War and the 1960s. The trends in age at marriage in Spain resembled those in other European countries but occurred somewhat later.

KEY woRDs: Marriage, Spain. 